\title{
Fractional exhaled nitric oxide-measuring devices: technology update
}

This article was published in the following Dove Press journal:

Medical Devices: Evidence and Research

23 June 2016

Number of times this article has been viewed

\author{
Mauro Maniscalco' \\ Carolina Vitale ${ }^{2}$ \\ Alessandro Vatrella ${ }^{2}$ \\ Antonio Molino ${ }^{3}$ \\ Andrea Bianco ${ }^{4}$ \\ Gennaro Mazzarella ${ }^{4}$ \\ 'Unit of Respiratory Diseases, \\ Hospital "S Maria della Pietà" of \\ Casoria, Naples, ${ }^{2}$ Unit of Respiratory \\ Medicine, Department of Medicine \\ and Surgery, University of Salerno, \\ Salerno, ${ }^{3}$ Department of Respiratory \\ Medicine, University Federico II, \\ ${ }^{4}$ Department of Cardiothoracic \\ and Respiratory Sciences, Second, \\ University of Naples, Naples, Italy
}

\begin{abstract}
The measurement of exhaled nitric oxide (NO) has been employed in the diagnosis of specific types of airway inflammation, guiding treatment monitoring by predicting and assessing response to anti-inflammatory therapy and monitoring for compliance and detecting relapse. Various techniques are currently used to analyze exhaled NO concentrations under a range of conditions for both health and disease. These include chemiluminescence and electrochemical sensor devices. The cost effectiveness and ability to achieve adequate flexibility in sensitivity and selectivity of NO measurement for these methods are evaluated alongside the potential for use of laser-based technology. This review explores the technologies involved in the measurement of exhaled NO.
\end{abstract}

Keywords: asthma, inflammation, nasal nitric oxide

\section{Introduction}

Since the identification of exhaled nitric oxide (NO) as a sensitive indicator of eosinophilic inflammation in the airways, its measurement has been employed in predicting and assessing a patient's response to anti-inflammatory therapy and monitoring compliance; ${ }^{1}$ accordingly, interest in developing new measurement techniques for NO concentrations has increased. ${ }^{2} \mathrm{NO}$ is a by-product of the oxidation of L-arginine to L-citrulline via three isoforms of synthase: two constitutively expressed NO synthases I and III (NOS1 and NOS3) and an inducibly expressed synthase II (NOS2). ${ }^{3}$ The complexity of the chemistry of $\mathrm{NO}$, a free radical molecule (containing an unpaired electron), with a short biological half-life (ranging from a few seconds to minutes), has been a major limitation when testing in pharmacological studies. ${ }^{4}$ In biological systems, NO is highly unstable and reacts with various compounds (eg, metals and thiol groups), and while more stable in air it reacts with oxygen $\left(\mathrm{O}_{2}\right)$ to form nitrogen dioxide $\left(\mathrm{NO}_{2}\right)$, a gas capable of inducing several types of tissue damage. ${ }^{5}$

\section{Exhaled NO measurement methods}

The diagnosis and management of airway inflammatory disease through NO concentration in exhaled breath has been driven by the technology available for its detection. ${ }^{6}$ Gustafsson et $\mathrm{al}^{7}$ first reported the detection of NO from human expired breath. To date, several techniques have been developed, such as chemiluminescence, electrochemical sensors, and laser-based technology (Figure 1), all of which present advantages and disadvantages in a clinical setting (Table 1).
Correspondence: Mauro Maniscalco Unit of Respiratory Diseases, Hospital "S Maria della Pietà" of Casoria, Largo delle Mimose I, 80I3I Naples, Italy

Tel +393381779679

Fax $+3981741 \quad 1457$

Email mauromaniscalco@hotmail.com 


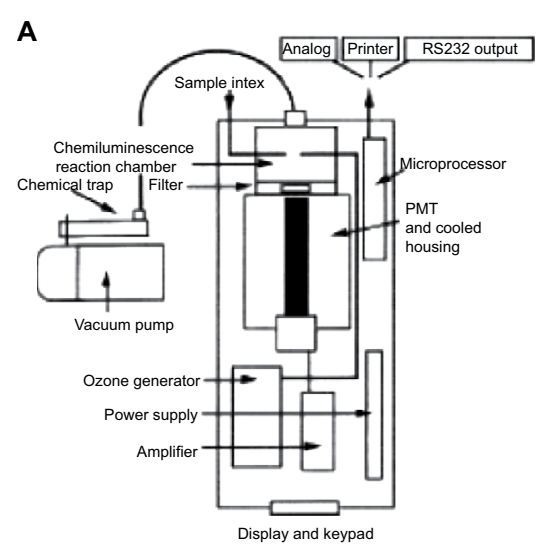

Chemiluminescence

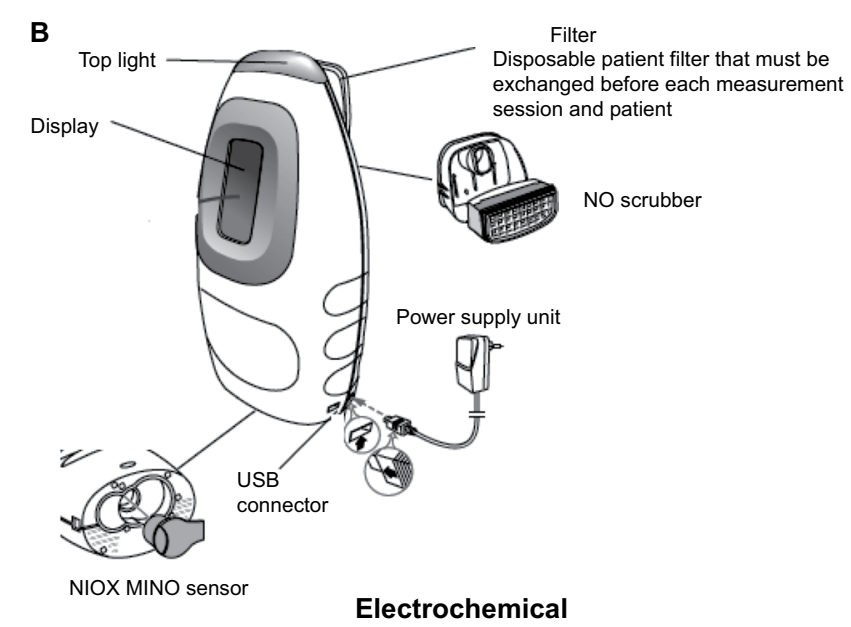

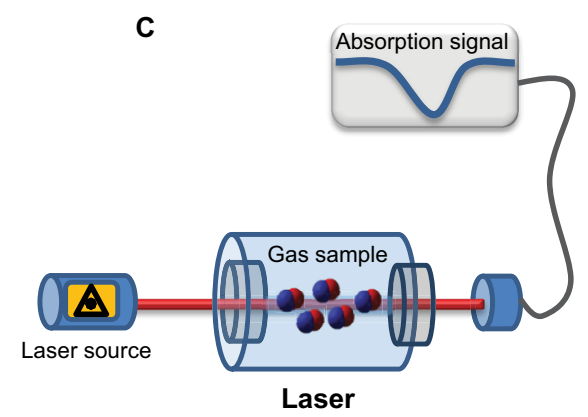

Figure I Schematic representation of a chemiluminescent analyzer (model 280i; Sievers) (A), an electrochemical sensor (NIOX MINO) (B), and a laser-based sensor (C). Abbreviations: NO, nitric oxide; PMT, photomultiplier tube.

Table I Performance characteristics of representative NO analyzers

\begin{tabular}{|c|c|c|c|}
\hline Characteristics & Chemiluminescence & Electrochemical & Laser \\
\hline Weight & $40 \mathrm{~kg}$ & $1 \mathrm{~kg}$ & $6-10 \mathrm{~kg}$ \\
\hline Sensitivity & $<\mathrm{lppb}$ & $>5 \mathrm{ppb}$ & I ppb \\
\hline Response time & $<$ I second & $>10$ seconds & I second \\
\hline $\begin{array}{l}\text { External } \\
\text { calibration }\end{array}$ & Yes & No & No \\
\hline Price & 50,000 EUR & 4,000 EUR & $>100,000$ EUR \\
\hline
\end{tabular}

Abbreviation: NO, nitric oxide.

\section{Chemiluminescence}

The chemiluminescence method represents the generally accepted "gold standard" method for gas phase NO analysis. NO molecules contained in the gas sample are detected on the basis of radiation created from their reaction with ozone $\left(\mathrm{O}_{3}\right)$. The $\mathrm{O}_{3}$ is generated in the instrument in vacuo, preventing contamination from other atoms, and reacts with the NO in an airstream sample. The reaction between $\mathrm{NO}$ and $\mathrm{O}_{3}$ generates nitrogen dioxide molecules $\left(\mathrm{NO}_{2}^{*}\right)$ in an electronically excited state. The subsequent reversion of these molecules to their lower energy ground state causes the emission of electromagnetic radiation (photons) with wavelengths ranging between $600 \mathrm{~nm}$ and 3,000 nm, which can in turn be detected and amplified by a photomultiplier tube. The resulting output signal is determined and corresponds linearly to the NO concentration in the sample, provided that $\mathrm{O}_{3}$ is present in excess.

Chemiluminescence equipment can be highly sensitive, with the detection threshold level at parts per billion (ppb; $1: 10^{9}$ ) and with very fast response times, between 0.5 seconds and 0.7 seconds. In addition, the technique permits direct analysis of the breath in situ or indirectly by sampling of the breath in a balloon that can be analyzed later. However, frequent calibration of the instrument is often required to ensure reliability and is achieved by using the concentration of NO up to hundreds of ppb. In addition, these analyzers need a source of external NO-free air to generate $\mathrm{O}_{3}$ within the equipment and a vacuum pump system, which raise manufacturing costs, with prices ranging between 20,000 USD and 45,000 USD. ${ }^{8}$ Furthermore, chemiluminescence analyzers are quite large, weighing between $25 \mathrm{~kg}$ and $45 \mathrm{~kg}$. These limitations have restricted the use of chemiluminescence analyzers in routine clinical applications or home monitoring and they currently remain in use solely for laboratory analysis.

Commercially available fractional exhaled nitric oxide (FENO) analyzers include NOA 280i (Sievers; GE Analytical Instruments, Boulder, CO, USA), NIOX (Aerocrine, Stockholm, Sweden), Logan model LR2149 (Logan Research 
Ltd., Rochester, UK), and CLD 88 (Eco Medics, Durnten, Switzerland).

\section{Standardization of device using chemiluminescence technology}

Methods for standardized and reproducible measurement of exhaled NO by chemiluminescence have been clarified. ${ }^{1}$ The first studies on exhaled NO using chemiluminescence analyzers presented large differences in values for healthy volunteers. These initial discrepancies were explained by the instrument's detection limits, response time, precision of calibration, and sampling methods, but in 1997, Silkoff et $\mathrm{al}^{9}$ showed that exhaled NO detection was affected by flow and it represented the main cause of variability.

The European Respiratory Society and American Thoracic Society have agreed on procedures for standardized measurements of lower respiratory tract exhaled NO. ${ }^{10}$ Recommendations by a European/North American joint taskforce were updated in 2005 in order to compare data from different groups. ${ }^{1}$ The importance of measuring $\mathrm{NO}$ at a flow rate of $50 \mathrm{~mL} / \mathrm{s}$, with the subject inhaling NO-free air, was established, while prolonged inhalation was not required. A restrictor facilitates an appropriate exhalation, achieving and maintaining $50 \mathrm{~mL} / \mathrm{s}$. A minimum airway pressure of $5 \mathrm{cmH}_{2} \mathrm{O}$ is required for the closure of the velum and to stop nasal NO contamination. A minimum of two NO plateau values within $10 \%$ of each other are required to be sampled. Further, NO measurements might be needed as a result of equipment variation and patient variability in patients' flow rate control. The mean NO concentration can then be accordingly evaluated.

Only a few studies have compared different types of chemiluminescent NO analyzers. In a study by Müller et al, ${ }^{11} \mathrm{NO}$ analyzers produced by different manufacturers (Aerocrine, Sievers, and Eco Medics) with varying setup and calibration regimes were compared. The authors found that the major factor responsible for variation in measured values between the NO analyzers was due to differences in calibration gases and concluded that analyzers from different manufacturers with a proper calibration showed adequate comparability for clinical purposes.

In another study, ${ }^{12}$ three NO analyzers (Eco Medics model CLD 88, NIOX, and Logan model LR2149) were compared in patients with asthma and chronic obstructive pulmonary disease (COPD) and healthy subjects. In this study, the authors found a relatively small, within analyzer, variability, although significantly greater variability between analyzers was detected. The group mean values obtained by the Logan analyzer were significantly higher, followed by the NIOX and then the Eco Medics analyzer. One explanation of these findings was that the differences observed may reflect variation in the flow rates between the analyzers.

\section{Electrochemical detection}

Electrochemical sensors have been employed for measuring exhaled NO. An electrochemical sensor converts gas concentration into electrical signals. Any gas that can be electrochemically oxidized or reduced can also be detected by means of an electrochemical sensor. The main measurement principle, adapted in the electrochemical sensor for NO analysis, is based on the amperometric technique, which is achieved in the electrochemical instrument by a buffer system that allows retention of the last portion of the exhalation sample. Subsequently, the sample is transferred to the sensor for analysis, where the target gas undergoes a chemical reaction in the presence of active catalytic sensor, and a measurable physical change is emitted within an electrical circuit. The sensor output signal, which presents a high sensitivity, is directly proportional to the partial pressure of $\mathrm{NO}$, and therefore to the concentration of NO, in the sample. The optimization of NO selectivity and sensitivity from the exhaled breath sample relies on catalyst and electrolyte composition with a complex arrangement of diffusion barrier membranes and a specific chemical filter system.

Several electrochemical sensor devices are commercially available: NIOX MINO and NIOX VERO (Aerocrine), NObreath (Bedfont Scientific Ltd, Maidstone, UK), and Hypair (Medisoft, Dinant, Belgium).

The NIOX MINO device is handheld and portable $(<1 \mathrm{~kg})$ and can be used by both adults and children. ${ }^{13}$ Patients have to produce a 10 -second exhalation of breath at an exhalation pressure of $10-20 \mathrm{cmH}_{2} \mathrm{O}$ in order to maintain a stable flow rate of $50 \pm 5 \mathrm{~mL} / \mathrm{s}$. A calibrated electrochemical sensor evaluates the final 3 seconds of exhalation expressing results in ppb with a range between $5 \mathrm{ppb}$ and $300 \mathrm{ppb}$. The NIOX MINO device is pre-calibrated and designed to ensure a service- and calibration-free system and utilizes a sensor which needs replacing between 100 and 300 measurements.

The NIOX VERO was developed to replace the NIOX MINO device. It is a battery-powered device featuring extended operational and test volume life compared to the NIOX MINO device, ${ }^{13}$ with a measurement range of 5-300 ppb.

The NObreath is a monitoring device, which requires 12 seconds of exhalation of breath in adults and 10 seconds in children, and weighs $\sim 400 \mathrm{~g}$ (including batteries); battery 
life lasts for up to 120 procedures. As sensor cells should be replaced every 2 years, set lifetime is not well defined. ${ }^{13}$

The Medisoft device is a semi-portable device (weighing $\sim 10 \mathrm{~kg}$ ) for repeatable measurement of exhaled NO with off-line measurement from an internal sample bag. It has a software package that provides step-by-step online quality control. The measurement range is $0-600 \mathrm{ppb}$. The NO cells are long lasting, typically 24 months or longer.

Recent advances in nano-engineered platforms have offered interesting technologies that can provide effective chemical interfaces for NO recognition. Madasamy et $\mathrm{al}^{14}$ have developed a virtual electrochemical user-friendly software that measures NO in exhaled breath from hydrogenperoxide-stimulated endothelial cells using an in-house manufactured potentiostat. It draws a linear plot and measures the concentration of NO present in the unknown sample. The data are acquired from the electrochemical oxidation of NO mediated by copper and zinc superoxide dismutase $(\mathrm{Cu}, \mathrm{ZnSOD})$. The electroanalytical results obtained were compared against the standard cyclic voltammetric instrument and were found to be in strong agreement. An important factor for this analyzer is its cost effectiveness and ability to achieve flexibility for sensitive and selective NO measurements.

A new electrochemical sensor device using the carbon allotrope, graphene, for measuring human exhaled NO concentration has been suggested. ${ }^{15}$ Graphene has a single atomic planar structure and its large surface area to volume ratio and high electron carrier mobility enable it to be a promising material for use as a conduction channel for both field-effect and chemical field-effect transistor sensors. Graphene-based field-effect transistors have been shown to detect individual NO gas molecules, and devices have been fabricated with alternating current dielectrophoresis. These devices offer high levels of sensitivity, recoverability, and reliable detection of NO gas (ranging from 2 ppb to $420 \mathrm{ppb}$ ), with a response time of several hundred seconds being achieved at room temperature. ${ }^{15}$ However, the main technological challenges for sensors used for measuring exhaled NO include factors such as the chemical or physical instability of both breath samples and sensor's elements and the variation in humidity and temperature. ${ }^{16,17}$

\section{Standardization of device using electrochemical technology}

Aerocrine developed the first electrochemical device for measuring fractional exhaled NO (NIOX MINO). ${ }^{18}$ Their initial studies on the reproducibility of this device were performed by comparison to the company's stationary device (NIOX). ${ }^{18-24}$ All these studies reported a close correlation between the two instruments, in both healthy controls and atopic (asthmatic and non-asthmatic) patients. Almost all the reported FENO values measured by the NIOX MINO were 1-2 ppb higher than those obtained by the stationary instrument, ${ }^{25}$ therefore, a correction equation to convert FENO values measured by NIOX MINO into that measured by the NIOX handheld device has been proposed. ${ }^{24}$

The Medisoft device measured exhaled NO concentrations higher than those of other analyzers. This difference was not consistent enough to enable the calculation of a similar correction factor. ${ }^{26}$ When using the NObreath device, the FENO values were consistently lower than the chemiluminescence analyzer. ${ }^{27}$ Similarly, NO values measured using the NIOX MINO were lower than those compared with the NOA280i (18.8 ppb vs 22.1 ppb). ${ }^{28}$

NIOX MINO was also compared with the Eco Medics stationary chemiluminescence $\mathrm{NO}$ analyzers, showing a good correlation and reproducibility of data. ${ }^{29}$ Korn et $\mathrm{al}^{26}$ have performed a comparative assessment of five different $\mathrm{NO}$ analyzers in patients affected by asthma and COPD and in normal controls during routine clinical practice: Medisoft, Aerocrine NIOX, Aerocrine NIOX flex, Aerocrine NIOX MINO, and Eco Medics analyzer CLD 88. Not all the NO analyzers tested demonstrated comparable results. While the median intra-individual differences between devices were of limited clinical relevance (1-8 ppb), the individual differences were as great as $150 \mathrm{ppb}$ in a single patient. Furthermore, the Bland-Altman plots indicated a good correlation of values only between devices produced by the same manufacturer (NIOX with NIOX flex and NIOX MINO). This was the case for asthmatic and COPD patients as well as normal controls, confirming that the variation between exhaled $\mathrm{NO}$ analyzers is independent of the presence and type of airway disease. ${ }^{30}$

Another factor was the long-term use of electrochemical sensors. Taylor et $\mathrm{al}^{31}$ found that the frequent use of devices affected the reproducibility of the measurements after 2 months of use with multiple sensor-analyzer combinations at baseline, although the difference $(<10 \mathrm{ppb})$ was classified as not relevant. In this study, the authors linked the problem to the lack of systematic calibration. The implication of these findings is that while in the main part these devices present a good correlation between FENO measurements, the equivalence of values could not necessarily be guaranteed in all conditions. ${ }^{13}$ Many studies have demonstrated that the measurements between devices are comparable within clinically acceptable limits, but a poorer 
equivalence has been reported between FENO devices at higher FENO levels. ${ }^{13}$ However, as the majority of studies show a high degree of correlation between measurements across all devices, the potential diagnostic accuracy of using FENO monitoring to guide diagnosis and management is likely, although the derived cutoff points are not likely to be interchangeable between devices. Therefore, if possible, serial measurements should be performed on individual patients, using the same type of analyzer, and the device used should be considered when interpreting the results.

The usefulness of the electrochemical device is based on its reliability and repeatability, as well as on its success in performing measurements from children. Test failure rates were very low for all devices in adults, with the highest reported rate being 3.3\%. However, for children using the NIOX MINO, a success rate of $84 \%$ was reported; ${ }^{22}$ in another study, the reproducibility of NIOX MINO measurements across the pediatric age range was poor when considering the absolute value of FENO, but acceptable when FENO values were categorized as low, normal, intermediate, or high. ${ }^{32} \mathrm{~A}$ further study reported no test failures using the NObreath device in children. In a comparative study in a mixed pediatric population, including normal, asthmatic, and allergic children using NIOX MINO and/or the NObreath devices, the FENO measurements with NIOX MINO demonstrated good repeatability, confirming manufacturer recommendation of obtaining only one test value. By contrast, the NObreath sensor required at least three exhalations, ${ }^{33}$ due to inadequacy and repeatability.

Recently, a new method for single-breath fraction of exhaled NO measurement for preschool children with asthma was proposed. ${ }^{34}$ This method was adapted by adjusting the exhalation time according to the child's age, and it was highly feasible down to the age of 4 years. A prototype instrument was used in this study, consisting of a handheld unit incorporating an electrochemical sensor and a newly developed flow control unit (NIOX NOVA; Aerocrine). The electrochemical sensor was validated by comparison with a chemiluminescence device (NIOX). Exhalation time was preadapted to age and set to 4 seconds, 6 seconds, $8 \mathrm{sec}$ onds, and 10 seconds, respectively, for the age of 3-4 years, 5-6 years, 7-8 years, and 9-10 years. An oval-shaped mouthpiece, used in nebulizers for small children, was used to aid maintenance of a tight seal. Of all the subjects, $>80 \%$ managed with at least one approved FENO measurement. One-quarter of the children aged 3 years, with no previous experience of FENO measurement or spirometry, succeeded with at least one measurement. Of all the subjects tested, $68 \%$ achieved at least two approved measurements, and the success rate improved with age, being $100 \%$ from the age of 8 years and above. ${ }^{34}$

\section{Laser-based technology}

Optical sensors based on different laser technologies and detection methods have been developed for the detection of NO concentrations. ${ }^{35}$ Schematically, these sensors include a laser source that produces light to interact with gas molecules, a gas cell containing the sample that has to be analyzed, and finally the detection system. For NO detection, the light source in the optical sensors must probe at the fundamental and strongest absorption band, centered in the mid-infrared (IR) region at $5.3 \mu \mathrm{m}$ ranging from $5.1 \mu \mathrm{m}$ to $5.7 \mu \mathrm{m}$.

Previously, the main limitation of the laser-based NO sensors was interference in this spectral range from several other gases, such as $\mathrm{CO}_{2}$ and $\mathrm{H}_{2} \mathrm{O}$. Hence, only specific absorption NO lines could be targeted, requiring only sensors that could generate the specific light spectra to be used. The first sensors employed which measured the absorption of the light were the tunable diode laser absorption spectroscopy, which could simultaneously detect $\mathrm{CO}$ and $\mathrm{NO}$ in the breath at a concentration of ppb. ${ }^{36}$ Unfortunately, these sensors required cooling to extremely low temperatures, prohibiting their use beyond the laboratory setting. Furthermore, the source was affected by spectral degradation. Similarly, another detection method, the Faraday rotation spectroscopy (FRS), which measures the rotation of the polarization of the light passing through a sample placed in a magnetic field, also required cooling to extremely low temperatures. ${ }^{37}$ Although this detection method was able to detect NO in breath analysis at very low concentrations, it was once again unable to be used outside the laboratory. A significant improvement in sensitivity of FRS method was achieved with the introduction of a heterodyne-enhanced FRS system (without any cryogenic cooling). ${ }^{38}$ Heterodyne detection techniques are based on the use of a local oscillator whose signal is combined with detected light. Beats between the local oscillator and the detected light are used to amplify the signal of interest, which can then be reconstructed by appropriate calculation. This allows significantly greater sensitivity than is available through direct detection. Differently from many spectroscopic systems limited by laser noise affecting the accuracy of the detection, this method provided significant noise reduction by employing heterodyne detection of the FRS signal. In fact, heterodyne-enhanced FRS shifts the FRS signal detection 
to radio frequency range, where the relative intensity noise of laser source is significantly lower. ${ }^{38}$

More recently, a new FRS-based method has been developed. This novel method relies on high frequency wavelength modulation of the laser with simultaneous modulation of the magnetic field. ${ }^{39}$ This dual modulation FRS method was tested in real clinical conditions to detect NO isotopes ( ${ }^{14} \mathrm{NO}$ and $\left.{ }^{15} \mathrm{NO}\right)$ in human breath and blood and urine samples. ${ }^{40}$

An improvement to the use of laser-based sensors was the fabrication of mid-IR lasers with a new semiconductor laser source, the quantum cascade laser (QCL). When first proposed in 1994, these worked only at cryogenic temperature, but QCLs are available sources for mid-IR wavelength functioning at room temperature. ${ }^{41}$ QCLs allow a sub-ppb detection of $\mathrm{NO}$ in $<1$ second.

A further development in NO detection was reached with the technology of high-finesse optical cavities. This system uses transparent dielectric mirrors characterized by a very high reflectivity that increases the optical path length, resulting in greater NO detection sensitivity. Based on this technology, different methods to detect NO were proposed such as cavity ring down spectroscopy, ${ }^{42}$ integrated cavity output spectroscopy, ${ }^{36}$ and cavity-enhanced absorption spectroscopy. ${ }^{43}$ These methods allow a sub-ppb detection of NO in 1 second and have been used to measure NO in breath with either off-line or online breath sample. In particular, cavity-enhanced absorption spectroscopy technology has been applied to the detection of breath biomarkers, and it has proven to be an effective tool for sensitive analysis of NO trace gas species in human breath. ${ }^{44}$

In comparison to the other spectrometric methods, highfinesse cavity-based systems are less affected by temperature variation. However, their use has been limited by the high technical skills required to ensure a careful alignment of the laser light, which is essential for an accurate NO detection. To increase the sensitivity of the detection of NO at low concentration, integrated detection systems have been proposed. For example, FRS sensors were integrated with QCLs that replaced $\mathrm{CO}$ lasers and allowed sub-ppb detection in $<1$ second. However, their use is limited by several technical problems, mainly related to the generation and shielding of the magnetic field..$^{45}$

Another example of integrated system with QCLs is offered by photoacoustic spectroscopy (PAS). This technique is based on the photoacoustic effect, whereby the gas of interest is intermittently irradiated by modulated light of a preselected wavelength. Subsequently, the light energy absorbed by the gas is converted into an acoustic signal which is detected by a microphone. The intensity of the generated sound is proportional to the light intensity. Hence, by measuring the amplitude of acoustic waves, photoacoustic detectors assess the concentration of gas.

A problem of photoacoustic detection of NO by use of QCLs is the high detection limit of NO (500 ppb), probably due to the lack of power in light intensity of QCLs on which the intensity of generated acoustic waves is dependent. ${ }^{46}$ Nevertheless, this problem has been recently overcome by a novel technique "quartz-enhanced PAS (QEPAS)", where the NO limit detection reached is 15 ppb within 5 seconds. ${ }^{36}$ The QEPAS sensor uses a quartz tuning fork as a sharply resonant acoustic transducer to detect weak photoacoustic excitation allowing the use of extremely small volumes. ${ }^{47}$ Differently from PAS, the NO limit detection reached by QEPAS is 15 ppb within 5 seconds.

\section{Standardization of device using laser-based technology}

The larger clinical studies into the detection of exhaled NO by laser-based sensors involved the use of two commercially available different systems. The first system is based on tunable diode laser absorption spectroscopy and was used to evaluate the distributions of exhaled NO in a wide population of 769 children and adults, allowing an NO detection limit of $1.5 \mathrm{ppb}$ in 4 seconds and an internal calibration. ${ }^{35}$

The second system was based on QCLs technology. Including two QCLs for multicomponent analysis (NO, CO, $\mathrm{CO}_{2}$, and $\mathrm{N}_{2} \mathrm{O}$ ), this instrument allowed a detection limit lower than $0.3 \mathrm{ppb}$ of $\mathrm{NO}$ within 1 second. It was used in several studies to analyze the breath biomarkers of COPD and asthma. ${ }^{48}$

In small clinical trials, other instruments based on QCLs were used and compared with the chemiluminescence and/ or electrochemical devices. In particular, in a population of 20 asthmatic children, the difference of the FENO values between the QCL-based sensor, chemiluminescent sensor, and the electrochemical sensor was found to be clinically acceptable. ${ }^{45}$

Over time, the clinical application of laser-based sensors has dealt with several limitations. The main limitation was that the development of this technology depended on the availability of the laser sources; furthermore, laser-based sensors have a very high cost, resulting in a poor commercial availability of laser-based detectors. 


\section{Other technologies}

Recently, new feasible techniques to measure exhaled NO have been developed; these include a method based on extractive electrospray ionization mass spectrometry for quantitative detection of exhaled $\mathrm{NO}$ at ppb level. ${ }^{49}$ FENO values are derived from the electrospray ionization mass spectrometry response of the product of selective reaction between 2-phenyl-4, 4, 5, 5-tetramethylimidazoline-1-oxyl3 -oxide reagent and $\mathrm{NO}$ molecules. However, the length of time required for sample collection and the usage of solvents hindered its application for online measurement. Dopant titrating ion mobility spectrometry has been utilized in breath analysis to measure exhaled NO. ${ }^{50}$ The time of 4 seconds for sample collection, however, represents a limiting factor for measuring the average exhalation FENO value and hampers the monitoring of FENO profile vs time, where a stable NO plateau can be exhibited.

Recently, a novel method based on fast nonequilibrium dilution ion mobility spectrometry was proposed to capture the exhaled NO profile in real time with response time of $75 \mathrm{~ms}$, while its profiles at different flow rates were obtained. ${ }^{51}$

\section{Nasal NO measurement methods}

The techniques discussed earlier have also been developed for nasal NO measurement, although it has been recognized as a diagnostic tool in a limited number of diseases..$^{52}$

Chemiluminescence represents the gold standard also for nasal NO measurements as these have a nasal NO device. Some recent studies have showed that nasal NO may also be measured by electrochemical device,,$^{53}$ demonstrating an acceptable accordance with the measurement obtained from the chemiluminescence analyzer. ${ }^{54-56}$

Current American Thoracic Society/European Respiratory Society guidelines recommend two methods for nasal NO measurements: ${ }^{1}$ nasal aspiration via one nostril with velum closure and nasal exhalation through a tight face mask with fixed flow. In the first method, nasal NO is aspirated from patients by the analyzer through a line with a disposable foam olive inserted into one nostril while the palate is closed by exhaling through the mouth into a disposable resistor (with a resistance of at least $10 \mathrm{cmH}_{2} \mathrm{O}$; Figure 2). Alternatively, with this method, nasal NO is aspirated while the subject holds breath with the velum elevated. In the second method, the nasal exhalation through a tight face mask with a stable fixed flow is used. In this case, the subjects start inhaling NOfree air from the analyzer through the nose and then exhale through a tightly fitting mask covering the nose connected to the analyzer.

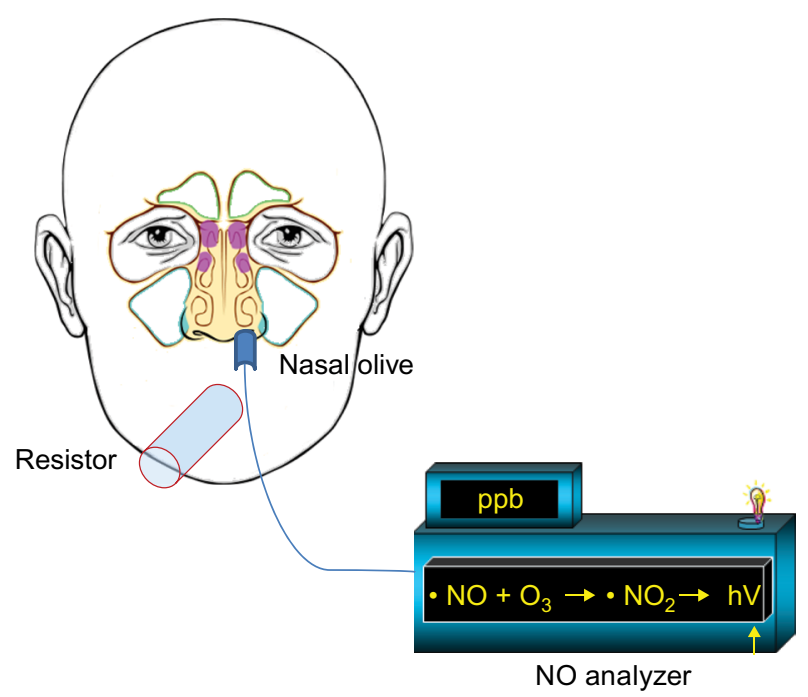

Figure 2. Recommended method for nasal NO measurement.

Notes: The NO is aspirated from the patient whilst soft palate is closed. This is done by exhalation through the mouth into a disposable resistor, using the analyzer through a line with a disposable foam olive inserted into one nostril.

Other possibilities for measuring nasal NO are using either nasal aspiration or nasal exhalation in a tight face mask during humming, ie, exhalation while phonating " $\mathrm{m}$ " without opening the lips or forming words, which is particularly useful to evaluate the obstruction of osteal meatal complex..$^{57,58}$ Both chemiluminescence and electrochemical sensors may be used for NO measurement during humming. ${ }^{59}$

\section{Measurement methods for extended $\mathrm{NO}$ analysis}

The measurement of exhaled NO at just one exhalation flow rate does not allow identification of NO production sites within the respiratory system. Therefore, mathematical models have been created to calculate the NO production within lung. The different models have been extensively reviewed by George et al and Högman et al. ${ }^{60,61}$

When the exhaled NO at different flow rates is detected in breath sampler, the NO production sites in the respiratory system can be calculated. In particular, the flux of NO from the airway wall to the lumen and fraction of NO in the gas phase alveolar region can be calculated when NO measurements are acquired at multiple high flow rates, while additional mathematical calculations with NO measurements obtained at both low and high flow rates can give the airway tissue concentration of $\mathrm{NO}$ released by the rigid conducting airway system and the transfer factor. Hence, more knowledge is gathered about the NO production sites of the respiratory system in the patient with the use of extended NO analysis. 
In all the chemiluminescence analyzers, expiratory flow rates can be modified by resistors, allowing an extended NO analysis. On the other hand, most of the electrochemical sensors are not suitable for multiple flow analysis. An exception is the Medisoft that allows assessing exhaled NO at multiple flow rates although only at high flow rates ranging from $50 \mathrm{~mL} / \mathrm{s}$ to $350 \mathrm{~mL} / \mathrm{s}$.

\section{Conclusion}

Different technologies for measurement of exhaled NO are available, and many easy-to-use NO analyzers are now accessible. Currently, chemiluminescence instruments are fast, sensitive, and highly selective for NO measurements. However, their expense, non-portability, and the necessity for frequent calibration limit their use in a clinical research setting. Electrochemical devices have demonstrated good correlation with a high level of precision and clinically accepted reproducibility to the "gold standard" chemiluminescence, with the advantages of portability and reduction in price. As such, these devices may have potential in the clinical routine management of inflammatory airways, although absolute exhaled NO measurements may vary depending on device employed; most of these are not suitable for multiple flow analysis. Finally, laser-based sensors have been used for clinical study to override the disadvantages of the two abovementioned technologies. In spite of high selectivity to the target compounds and fast response time, expensive cost, spectral degradation, and reliability problems of laser source represent limiting factors for wide clinical use.

All the new sensors used for exhaled NO might be affected by factors such as the variation of humidity and temperature, which may interfere with stable analysis of real-world breath sampling. ${ }^{62}$ These factors might also be responsible for differences observed in NO measurements among different analyzers and category of device. ${ }^{16}$

New technologies are in development, which should allow more appropriate exploitation of NO exhalation levels for the diagnosis, treatment, and monitoring of relevant respiratory disorders.

\section{Disclosure}

The authors report no conflicts of interest in this work.

\section{References}

1. American Thoracic Society, European Respiratory Society. ATS/ERS recommendations for standardized procedures for the online and offline measurement of exhaled lower respiratory nitric oxide and nasal nitric oxide, 2005. Am J Respir Crit Care Med. 2005;171(8):912-930.

2. Barnes PJ, Dweik RA, Gelb AF, et al. Exhaled nitric oxide in pulmonary diseases: a comprehensive review. Chest. 2010;138(3):682-692.
3. Ricciardolo FL, Sterk PJ, Gaston B, Folkerts G. Nitric oxide in health and disease of the respiratory system. Physiol Rev. 2004;84(3):731-765.

4. Nathan C, Xie QW. Nitric oxide synthases: roles, tolls, and controls. Cell. 1994;78(6):915-918.

5. Grisham MB, Jourd'Heuil D, Wink DA. Nitric oxide. I. Physiological chemistry of nitric oxide and its metabolites:implications in inflammation. Am J Physiol. 1999;276(2 Pt 1):G315-G321.

6. Dweik RA, Boggs PB, Erzurum SC, et al; American Thoracic Society Committee on Interpretation of Exhaled Nitric Oxide Levels (FENO) for Clinical Applications. An official ATS clinical practice guideline: interpretation of exhaled nitric oxide levels (FENO) for clinical applications. Am J Respir Crit Care Med. 2011;184(5):602-615.

7. Gustafsson LE, Leone AM, Persson MG, Wiklund NP, Moncada S. Endogenous nitric oxide is present in the exhaled air of rabbits, guinea pigs and humans. Biochem Biophys Res Commun. 1991;181(2): 852-857.

8. Maniscalco M, Lundberg JO. Hand-held nitric oxide sensor NIOX $\mathrm{MINO}(\mathrm{R})$ for the monitoring of respiratory disorders. Expert Rev Respir Med. 2010;4(6):715-721.

9. Silkoff PE, McClean PA, Slutsky AS, et al. Marked flow-dependence of exhaled nitric oxide using a new technique to exclude nasal nitric oxide. Am J Respir Crit Care Med. 1997;155(1):260-267.

10. Kharitonov S, Alving K, Barnes PJ. Exhaled and nasal nitric oxide measurements: recommendations. The European Respiratory Society Task Force. Eur Respir J. 1997;10(7):1683-1693.

11. Müller KC, Jörres RA, Magnussen H, Holz O. Comparison of exhaled nitric oxide analysers. Respir Med. 2005;99(5):631-637.

12. Borrill Z, Clough D, Truman N, Morris J, Langley S, Singh D. A comparison of exhaled nitric oxide measurements performed using three different analysers. Respir Med. 2006;100(8):1392-1396.

13. Harnan SE, Tappenden P, Essat M, et al. Measurement of exhaled nitric oxide concentration in asthma: a systematic review and economic evaluation of NIOX MINO, NIOX VERO and NObreath. Health Technol Assess. 2015;19(82):1-330.

14. Madasamy T, Pandiaraj M, Balamurugan M, et al. Virtual electrochemical nitric oxide analyzer using copper, zinc superoxide dismutase immobilized on carbon nanotubes in polypyrrole matrix. Talanta. 2012;100:168-174.

15. Li W, Geng X, Guo Y, et al. Reduced graphene oxide electrically contacted graphene sensor for highly sensitive nitric oxide detection. ACS Nano. 2011;5(9):6955-6961.

16. Konvalina G, Haick H. Sensors for breath testing: from nanomaterials to comprehensive disease detection. Acc Chem Res. 2014;47(1):66-76.

17. Konvalina G, Haick H. Effect of humidity on nanoparticle-based chemiresistors: a comparison between synthetic and real-world samples. ACS Appl Mater Interfaces. 2012;4(1):317-325.

18. Hemmingsson T, Linnarsson D, Gambert R. Novel hand-held device for exhaled nitric oxide-analysis in research and clinical applications. J Clin Monit Comput. 2004;18(5-6):379-387.

19. Alving K, Janson C, Nordvall L. Performance of a new hand-held device for exhaled nitric oxide measurement in adults and children. Respir Res. 2006;7:67.

20. McGill C, Malik G, Turner SW. Validation of a hand-held exhaled nitric oxide analyzer for use in children. Pediatr Pulmonol. 2006;41(11): 1053-1057.

21. Vahlkvist S, Sinding M, Skamstrup K, Bisgaard H. Daily home measurements of exhaled nitric oxide in asthmatic children during natural birch pollen exposure. J Allergy Clin Immunol. 2006;117(6):1272-1276.

22. Khalili B, Boggs PB, Bahna SL. Reliability of a new hand-held device for the measurement of exhaled nitric oxide. Allergy. 2007;62(10): 1171-1174.

23. Menzies D, Nair A, Lipworth BJ. Portable exhaled nitric oxide measurement: comparison with the "gold standard" technique. Chest. 2007; 131(2):410-414.

24. Pizzimenti S, Bugiani M, Piccioni P, et al. Exhaled nitric oxide measurements: correction equation to compare hand-held device to stationary analyzer. Respir Med. 2008;102(9):1272-1275. 
25. Chen W, Purohit A, Barnig C, et al. Niox and Niox Mino: comparison of exhaled NO in grass pollen allergic adult volunteers. Allergy. 2007; 62(5):571-572.

26. Korn S, Telke I, Kornmann O, Buhl R. Measurement of exhaled nitric oxide: comparison of different analysers. Respirology. 2010; 15(8):1203-1208.

27. Pisi R, Aiello M, Tzani P, Marangio E, Olivieri D, Chetta A. Measurement of fractional exhaled nitric oxide by a new portable device: comparison with the standard technique. J Asthma. 2010;47(7):805-809.

28. Kim SH, Moon JY, Kwak HJ, et al. Comparison of two exhaled nitric oxide analyzers: the NIOX MINO hand-held electrochemical analyzer and the NOA280i stationary chemiluminescence analyzer. Respirology. 2012;17(5):830-834.

29. Boot JD, de Ridder L, de Kam ML, Calderon C, Mascelli MA, Diamant Z. Comparison of exhaled nitric oxide measurements between NIOX MINO electrochemical and Ecomedics chemiluminescence analyzer. Respir Med. 2008;102(11):1667-1671.

30. Schiller B, Hammer J, Barben J, Trachsel D. Comparability of a hand-held nitric oxide analyser with online and offline chemiluminescence-based nitric oxide measurement. Pediatr Allergy Immunol. 2009;20(7):679-685.

31. Taylor DR, Palmay R, Cowan JO, Herbison GP. Long term performance characteristics of an electrochemical nitric oxide analyser. Respir Med. 2011;105(2):211-217.

32. Selby A, Clayton B, Grundy J, et al. Are exhaled nitric oxide measurements using the portable NIOX MINO repeatable? Respir Res. 2010;11:43.

33. Kapande KM, McConaghy LA, Douglas I, et al. Comparative repeatability of two handheld fractional exhaled nitric oxide monitors. Pediatr Pulmonol. 2012;47(6):546-550.

34. Heijkenskjöld-Rentzhog C, Kalm-Stephens P, Nordvall L, Malinovschi A, Alving K. New method for single-breath fraction of exhaled nitric oxide measurement with improved feasibility in preschool children with asthma. Pediatr Allergy Immunol. 2015;26(7):662-667.

35. Wang C, Sahay P. Breath analysis using laser spectroscopic techniques breath biomarkers, spectral fingerprints, and detection limits. Sensors (Basel). 2009;9(10):8230-8262.

36. Bakhirkin YA, Kosterev AA, Roller C, Curl RF, Tittel FK. Midinfrared quantum cascade laser based off-axis integrated cavity output spectroscopy for biogenic nitric oxide detection. Appl Opt. 2004;43(11):2257-2266

37. Cristescu SM, Mandon J, Harren FJ, Meriläinen P, Högman M. Methods of NO detection in exhaled breath. J Breath Res. 2013;7(1):017104.

38. Wang Y, Nikodem M, Wysocki G. Cryogen-free heterodyne-enhanced mid-infrared Faraday rotation spectrometer. Opt Express. 2013; 21(1):740-755.

39. Brumfield B, Sun W, Wang Y, Ju Y, Wysocki G. Dual modulation Faraday rotation spectroscopy of $\mathrm{HO} 2$ in a flow reactor. Opt Lett. 2014; 39(7):1783-1786.

40. Wang Y, Nikodem M, Zhang E, et al. Shot-noise limited Faraday rotation spectroscopy for detection of nitric oxide isotopes in breath, urine, and blood. Sci Rep. 2015;5:9096.

41. Faist J, Capasso F, Sivco DL, Sirtori C, Hutchinson AL, Cho AY. Quantum cascade laser. Science. 1994;264(5158):553-556.

42. Kosterev AA, Malinovsky AL, Tittel FK, et al. Cavity ringdown spectroscopic detection of nitric oxide with a continuous-wave quantumcascade laser. Appl Opt. 2001;40(30):5522-5529.
43. Menzel L, Kosterev AA, Curl RF, et al. Spectroscopic detection of biological NO with a quantum cascade laser. Appl Phys B. 2001; 72(7):859-863.

44. Wojtas J. Application of cavity enhanced absorption spectroscopy to the detection of nitric oxide, carbonyl sulphide, and ethane - breath biomarkers of serious diseases. Sensors (Basel). 2015;15(6):14356-14369.

45. Mandon J, Högman M, Merkus PJ, van Amsterdam J, Harren FJ, Cristescu SM. Exhaled nitric oxide monitoring by quantum cascade laser: comparison with chemiluminescent and electrochemical sensors. $J$ Biomed Opt. 2012;17(1):017003.

46. Elia A, Lugara PM, Giancaspro C. Photoacoustic detection of nitric oxide by use of a quantum-cascade laser. Opt Lett. 2005;30(9):988-990.

47. Patimisco P, Scamarcio G, Tittel FK, Spagnolo V. Quartz-enhanced photoacoustic spectroscopy: a review. Sensors (Basel). 2014;14(4):6165-6206.

48. Shorter JH, Nelson DD, McManus JB, Zahniser MS, Sama SR, Milton DK. Clinical study of multiple breath biomarkers of asthma and COPD (NO, CO(2), CO and $\mathrm{N}(2) \mathrm{O})$ by infrared laser spectroscopy. J Breath Res. 2011;5(3):037108.

49. Pan S, Tian Y, Li M, et al. Quantitative detection of nitric oxide in exhaled human breath by extractive electrospray ionization mass spectrometry. Sci Rep. 2015;5:8725.

50. Peng L, Hua L, Li E, et al. Dopant titrating ion mobility spectrometry for trace exhaled nitric oxide detection. J Breath Res. 2015;9(1):016003.

51. Peng L, Jiang D, Wang Z, Liu J, Li H. Online measurement of exhaled NO concentration and its production sites by fast non-equilibrium dilution ion mobility spectrometry. Sci Rep. 2016;6:23095.

52. Maniscalco M, Sofia M, Pelaia G. Nitric oxide in upper airways inflammatory diseases. Inflamm Res. 2007;56(2):58-69.

53. Weschta M, Deutschle T, Riechelmann H. Nasal fractional exhaled nitric oxide analysis with a novel hand-held device. Rhinology. 2008;46(1):23-27.

54. Montella S, Alving K, Maniscalco M, et al. Measurement of nasal nitric oxide by hand-held and stationary devices. Eur J Clin Invest. 2011;41(10): 1063-1070.

55. Marthin JK, Nielsen KG. Hand-held tidal breathing nasal nitric oxide measurement - a promising targeted case-finding tool for the diagnosis of primary ciliary dyskinesia. PLoS One. 2013;8(2):e57262.

56. Maniscalco M, de Laurentiis G, Weitzberg E, Lundberg JO, Sofia M. Validation study of nasal nitric oxide measurements using a hand-held electrochemical analyser. Eur J Clin Invest. 2008;38(3):197-200.

57. Maniscalco M, Weitzberg E, Sundberg J, Sofia M, Lundberg JO. Assessment of nasal and sinus nitric oxide output using single-breath humming exhalations. Eur Respir J. 2003;22(2):323-329.

58. Maniscalco M, Sofia M, Weitzberg E, et al. Humming-induced release of nasal nitric oxide for assessment of sinus obstruction in allergic rhinitis: pilot study. Eur J Clin Invest. 2004;34(8):555-560.

59. Maniscalco M, Pelaia G, Sofia M. Exhaled nasal nitric oxide during humming: potential clinical tool in sinonasal disease? Biomark Med. 2013;7(2):261-266.

60. George SC, Hogman M, Permutt S, et al. Modeling pulmonary nitric oxide exchange. J Appl Physiol (1985). 2004;96(3):831-839.

61. Högman M, Malinovschi A, Norbäck D, Janson C. Added value with extended NO analysis in atopy and asthma. Clin Physiol Funct Imaging. 2011;31(4):294-299.

62. Mathew TL, Pownraj P, Abdulla S, Pullithadathil B. Technologies for clinical diagnosis using expired human breath analysis. Diagnostics (Basel). 2015;5(1):27-60. 


\section{Publish your work in this journal}

Medical Devices: Evidence and Research is an international, peerreviewed, open access journal that focuses on the evidence, technology, research, and expert opinion supporting the use and application of medical devices in the diagnosis, monitoring, treatment and management of clinical conditions and physiological processes. The identification of novel devices and optimal use of existing devices which will lead to improved clinical outcomes and more effective patient management and safety is a key feature. The manuscript management system is completely online and includes a quick and fair peer-review system. Visit http://www. dovepress.com/testimonials.php to read real quotes from authors.

Submit your manuscript here: https://www.dovepress.com/medical-devices-evidence-and-research-journal 